Original article

\title{
Role of Latex Particle Agglutination Test in the Diagnosis of Meningitis
}

\author{
Nurjahan Begum, Iftikhar Ahmed, Md. Abdus Salam, Shahanara Begum, KM Faisal Alam \\ Department of Microbiology, Rajshahi Medical College, Rajshahi
}

\begin{abstract}
A total of 45 clinically suspected cases of meningitis of all ages, admitted at Rajshahi Medical College Hospital (RMCH), were studied to find out the etiological agents of meningitis. Among the cases, 32 (71.1\%) were diagnosed as aseptic meningitis and $13(28.89 \%)$ as bacterial meningitis by the conventional CSF study. But out of total 45 cases, $18(40 \%)$ were diagnosed as bacterial meningitis by Latex particle agglutination test (LPAT). The LPAT is widely used in clinical microbiology laboratories and can be considered as an important supplement to the culture and Gram stain of CSF specimens. Rapid antigen detection tests may provide positive results when culture and Gram stain results are negative for meningitis patients who have received antimicrobial therapy. So, in our country, where empirical broad-spectrum antibiotic is randomly used, LPAT may be considered as an adjunct to establish the diagnosis of bacterial meningitis
\end{abstract}

Key words: Bacterial meningitis, CSF, Latex particle agglutination test (LPAT)

\section{Introduction}

Meningitis constitutes to be a life-threatening infection throughout the world. It refers to an inflammatory process of the leptomeninges and CSF within subarachnoid space of the brain and spinal cord and the ventricular system. ${ }^{1}$ The syndrome of meningitis may be caused by a number of etiological agents. There are two predominant types of meningitis such as bacterial and viral, based on causative agents. ${ }^{2}$ Epidemiological pattern of meningitis varies greatly from country to country. Bacterial meningitis is a major cause of death and disability in children worldwide. More than one million cases with 200,000 deaths have been estimated to occur each year due to bacterial meningitis. ${ }^{3}$ Early etiological diagnosis and appropriate treatment of meningitis cases result in a higher cure rate and lower incidence of potentially fatal complications. ${ }^{4}$ To meet up the acute situation in the management of meningitis cases, sensitive and rapid diagnostic methods are needed. The detection of bacterial

Correspondence:

Dr. Nurjahan Begum, Clinical Pathologist

Department of Microbiology, Rajshahi Medical College

Rajshahi. antigens in CSF could be an important diagnostic tool for early empirical antibiotic therapy in bacterial meningitis. ${ }^{5}$

Antigens of Haemophilus influenzae type b, Streptococcus pneumoniae, Neisseria meningitidis, Streptococcus agalactiae and Escherichia coli in CSF can be detected by Latex particle agglutination test (LPAT). It is very useful in the diagnosis of bacterial meningitis with sensitivity and specificity ranging from $95 \%$ to $100 \%$. The LPAT is simple to perform and laboratory technicians are also able to interpret the results of the tests. It requires minimum time and the use of antibiotics generally do not alter the results when used for short intervals. ${ }^{6}$

We carried out a study to assess the clinical usefulness of the Latex agglutination test in patients diagnosed of having meningitis, focusing on the spectrum of causative bacteria identified by this method.

\section{Methods}

All cases of meningitis, diagnosed in Medicine and Pediatric units of Rajshahi Medical College Hospital between January, 2006 and July, 2006, were analyzed. We reviewed all medical 
records and data collected including age, sex, clinical features, results of CSF analysis. Rapid qualitative latex agglutination techniques were used to detect specific polysaccharide surface antigens for $H$. Influenzae type $\mathrm{b}$ (Hib), S. pneumoniae, E. coli, group B Streptococcus and $N$. meningitidis A, C, Y, W-135, and B by Slidex Minengite-Kits of France. The CSF study was done for each patient by conventional method and data were analyzed following standard methods.

\section{Results}

A total 45 cases of meningitis were diagnosed during this study period of which $32(71.7 \%)$ were found aseptic from all age groups. Majority of the patients were from poor socioeconomic condition.

Common presenting complaints were fever, headache, vomiting, altered consciousness. Neck rigidity and Kerning's sign were positive in $84.4 \%$ and $95.5 \%$ cases respectively. Some $34(75.5 \%)$ of the patients had received antibiotics prior to hospitalization. Results of Gram stain were found positive in $6(13.3 \%)$ cases. But the LPAT for bacterial antigen were positive in $18(40 \%)$ out of total 45 cases. (Table I)

Table I: Latex particle agglutination tests for soluble antigens

\begin{tabular}{llr}
\hline $\begin{array}{l}\text { Bacterial antigens tested for } \\
(\mathrm{n}=45)\end{array}$ & $\begin{array}{l}\text { Positive } \\
\text { Number }\end{array}$ & $(\%)$ \\
\hline H. influenzae & 08 & 44.44 \\
N. meningitidis & 03 & 16.67 \\
S. pneumoniae & 06 & 33.33 \\
Streptococcus group B & 00 & 00 \\
N. meningitidis B/E. coli & 01 & 5.56 \\
Total & 18 & 100.00 \\
\hline
\end{tabular}

\section{Discussion}

In acute bacterial meningitis, the classic symptoms and signs of meningeal irritation are common, but these signs may be present in other diseases like acute viral meningitis, tuberculous meningitis, subarachnoid hemorrhage, etc. Most of the patients of acute meningitis usually receive broadspectrum antimicrobial therapy before any diagnostic approach taken. The CSF should be examined in every patient in whom the clinical findings are consistent with the possibility of meningitis. Alternative methods of CSF study have been developed which may be useful in patients commenced with antibiotic therapy before lumber puncture. Where culture is usually negative, detection of soluble bacterial antigens can help to reach a diagnosis. The Latex Particle Agglutination Test (LPAT) has been introduced for this purpose because it can detect comparatively very small quantity of antigens present.

Although specificity of these tests is good, sensitivity is not better than a Gram stain. Therefore, negative results for a specific bacterial antigen do not rule out bacterial meningitis. ${ }^{7}$

The main limitation of LPAT is that it is positive only in the presence of specific polysaccharide surface antigens for $H$. influenzae type b (Hib), S. pneumoniae, E. coli, group B Streptococcus and N. meningitidis A, C,Y, W-135 antigens, while any other bacteria remain undetected. The Gram's staining method do not have this limitation. To optimize cost benefit ratio, the test should not be indiscriminately used. Thus, the LAPT should be done routinely when positive Gram stain and abnormal CSF values (WBC counts, glucose, protein concentrations and other markers) indicated bacterial meningitis.

The antigens of common meningeal pathogens e.g. $H$. influenzae type b (Hib), S. pneumoniae, E. coli, group B Streptococcus and $N$. meningitidis are detected by the LPAT. The diagnosis of bacterial meningitis caused by specific pathogens are established by positive LPAT, but a negative test does not rule out other bacterial meningitis cases. A broad-spectrum antibiotic coverage is usually recommended as an initial treatment for suspected bacterial meningitis, which is very costly. The LPAT can diagnose these specific bacterial pathogens and specific antibiotic therapy can be given to reduce the emergence of bacterial resistance.

\section{References}

1. Crook DW, Phupradit P, Warrel DA. Bacterial Meningitis. In: Weatherall D, Ledingharn JG, Warrell DA, editors. Oxford Test book of Medicine, 3rd ed. New York: Oxford University Press Inc; 1996: pp. 4050-4064.

2. Hicks R, Macnair T. Meningitis. BBC Health. Web page at "http://www.meningitistrust. org" [accessed on 26.12.2006]

3. Seyoum MDG. Haemophilus influenzae type $\mathrm{b}$ meningitis in children in Eritrea. Emerg Infect 2004; 10 (1): 30-32.

4. Bijoy R, Mirdha U, Gupta, Bhujwala RA. Latex agglutination test: An adjunct to the laboratory diagnosis of pyogenic bacterial meningitis. Indian J Paediatr 1991; 58: 521-524.

5. Das BK, Gurbacharya RL, Mohapatra TK, Mishra OP. Bacterial 
antigen detection test in meningitis. Indian J Paed 2003; 70: 779780.

6. Camargosp PAM, Almeida, MS, Cardoso. Latex Particle agglutination test in diagnosis of Haemophilus influaenzae type b, Streptococcus Pneumoniae and Neisseria meningitidis A and
C meningitis in infants and children. J Clin Epidemiol 1995; 48: 1245-1250.

7. Phillips EJ, Simor AR. Bacterial meningitis in children and adults. Post Graduate Med 1998; 130 (3): 1-12. 